\title{
Crohn's Disease in which the Patient Developed Aortitis during Treatment with Adalimumab
}

\author{
Hiroki Kiyohara ${ }^{1}$, Tadakazu Hisamatsu ${ }^{1}$, Katsuyoshi Matsuoka ${ }^{1}$, Makoto Naganuma ${ }^{2}$, \\ Hideto Kameda ${ }^{3,4}$, Noriyuki Seta ${ }^{3}$, Tsutomu Takeuchi ${ }^{3}$ and Takanori Kanai ${ }^{1}$
}

\begin{abstract}
A 23-year-old woman developed aortitis during treatment with adalimumab (ADA) for ileocolic Crohn's disease (CD). The patient complained of a high fever, abdominal pain, diarrhea, hematochezia and arthralgia. Although the ADA therapy resulted in immediate symptom improvement, after six weeks, she again complained of a low-grade fever and abdominal pain, whereas the CD-related symptoms did not worsen. Contrast-enhanced computed tomography revealed thoracoabdominal aortitis, and we therefore started treatment with prednisolone, which immediately improved the fever and abdominal pain. We subsequently tapered the dose of prednisolone and resumed the administration of ADA in order to maintain the CD remission. No further episodes of aortitis relapse were noted after restarting ADA, and the CD currently remains in remission. This is the first report of the onset of aortitis during ADA therapy for CD.
\end{abstract}

Key words: crohn's disease, aortitis, adalimumab

(Intern Med 54: 1725-1732, 2015)

(DOI: 10.2169/internalmedicine.54.3853)

\section{Introduction}

Crohn's disease (CD) is a chronic inflammatory disorder of the gastrointestinal tract characterized by the presence of transmural inflammation with granulomatous changes and characteristic endoscopic findings of longitudinal ulcers or 'skip lesions.' CD sometimes presents with extraintestinal complications, including arthritis, uveitis and erythema nodosum. Takayasu arteritis (TA) is a rare granulomatous vasculitis involving the aorta and its major branches. These are chronic inflammatory diseases considered to be immunological disorders. However, the mechanisms underlying the pathogenesis of these conditions remains unknown.

Although the prevalence of $\mathrm{CD}$ and TA is estimated to be 1 per 10 billion, cases involving the concurrent onset of both diseases have been reported (1-4). It is likely that the pathogenesis of $\mathrm{CD}$ is related in some way to that of TA, although this phenomenon is not yet well understood.

Anti-tumor necrosis factor (TNF)- $\alpha$ agents are used to treat both $\mathrm{TA}$ and $\mathrm{CD}$, although some patients with $\mathrm{CD}$ paradoxically develop TA during treatment with anti-TNF- $\alpha$ drugs (5-7). We herein report the case of a patient with $\mathrm{CD}$ who developed aortitis, consistent with the features of TA, despite receiving treatment with adalimumab (ADA) for CD.

\section{Case Report}

A 23-year-old Japanese woman visited a community hospital with a high fever, abdominal pain, diarrhea, arthralgia and hematochezia. The patient had a past medical history of uveitis, which had become quiescent. She had no family history of inflammatory bowel disease. Although she had received no medications when she first visited the hospital, total colonoscopy was subsequently performed, which revealed erosions and longitudinal ulcers with skip lesions in the colon (Fig. 1A). Based on these findings and her past medical history of uveitis, CD was suspected. Hence, the administration of mesalazine was started at a daily dose of 3 $\mathrm{g}$, with continued fasting under treatment with total par-

\footnotetext{
${ }^{1}$ Division of Gastroenterology and Hepatology, Department of Internal Medicine, Keio University School of Medicine, Japan, ${ }^{2}$ Center for Diagnostic and Therapeutic Endoscopy, Keio University School of Medicine, Japan, ${ }^{3}$ Division of Rheumatology, Department of Internal Medicine, Keio University School of Medicine, Japan and ${ }^{4}$ Division of Rheumatology, Toho University Ohashi Medical Center, Japan Received for publication August 14, 2014; Accepted for publication November 18, 2014 Correspondence to Dr. Tadakazu Hisamatsu, hisamachi@a7.keio.jp
} 

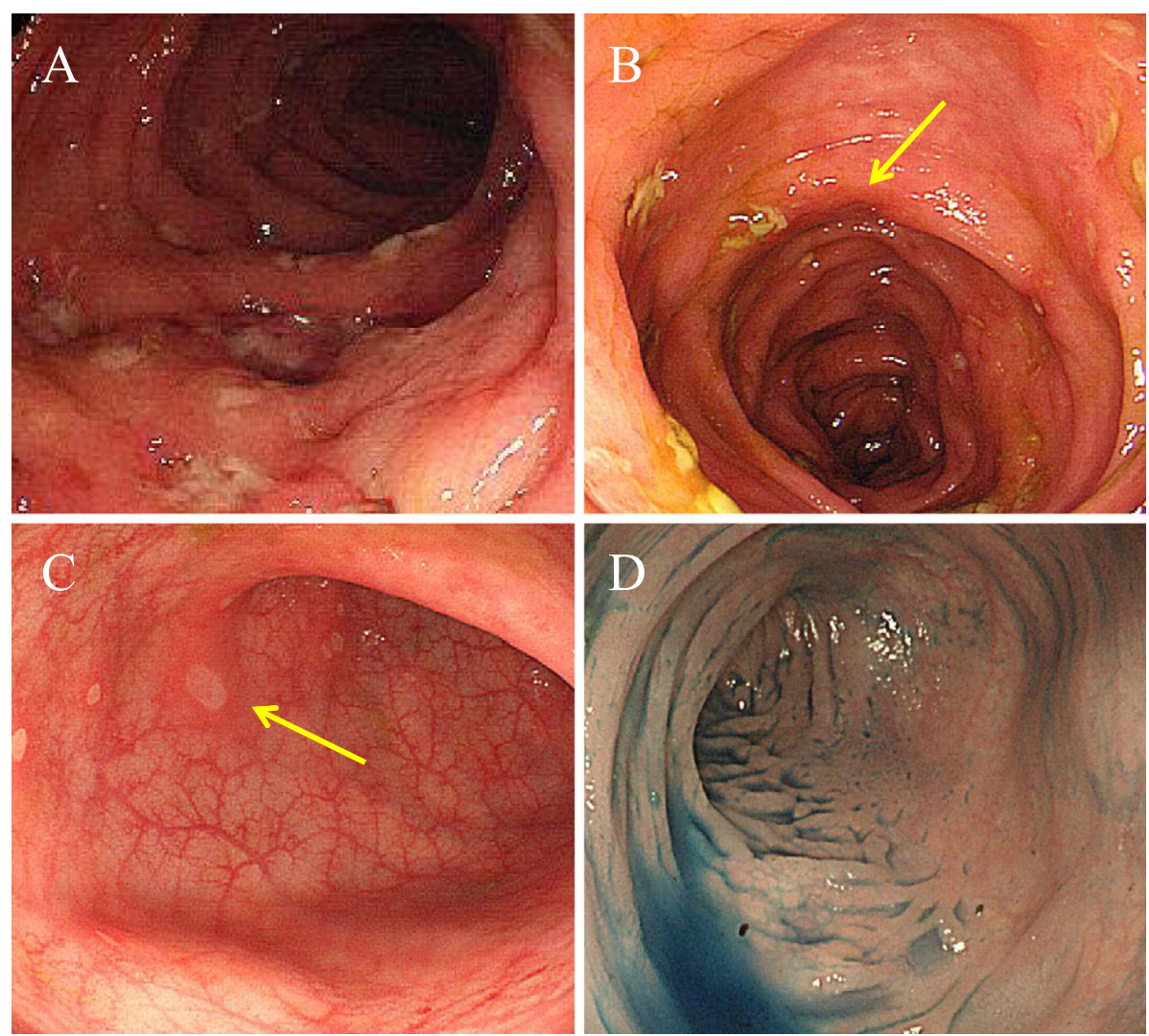

Figure 1. Total colonoscopy performed before and after treatment with ADA. (A) Prior to the initiation of ADA therapy, erosions and ulcers with skip lesions were detected, indicative of a diagnosis of CD. (B, C) Aphthous erosions were aligned longitudinally, suggesting CD (arrows). (D) Total colonoscopy performed eight months after the first administration of ADA. Ulcer scars were noted in the transverse and descending colon, tending to localize on one side of the wall. The lesions were also distributed discontinuously, with some of them arranged longitudinally. These findings can be regarded as indicating endoscopic remission.

enteral nutrition. The patient was subsequently referred to our hospital and admitted.

Upon admission to our hospital, the patient complained of a high fever, polyarthralgia and muddy stools. The mean frequency of defecation was approximately four times daily; however, a stool culture showed no causative microorganisms. A physical examination conducted on admission disclosed swelling and tenderness of the ankle joints bilaterally, and indolent erythema was noted on the dorsum of the right foot. One week after admission, another indolent erythematous lesion emerged on the left foot. We believed these lesions to be atypical of erythema nodosum due to the absence of pain and speculated that they may be extraintestinal manifestations of CD.

Laboratory data obtained on admission demonstrated an increased white blood cell count (WBC: 14,200/ $\mu \mathrm{L}$ ), platelet count $\left(41.8 \times 10^{4} / \mu \mathrm{L}\right)$, C-reactive protein level (CRP: 8.68 $\mathrm{mg} / \mathrm{dL})$ and erythrocyte sedimentation rate $(135 \mathrm{~mm} / \mathrm{h})$ with normocytic anemia (hemoglobin: $7.8 \mathrm{~g} / \mathrm{dL}$, mean corpuscular volume, MCV: $86 \mathrm{fL}$ ) (Table 1). In addition, laboratory tests also showed decreased serum total protein $(5.5 \mathrm{~g} / \mathrm{dL})$, albumin $(2.3 \mathrm{~g} / \mathrm{dL})$ and total cholesterol $(86 \mathrm{mg} / \mathrm{dL})$ levels. Furthermore, the serum iron level was normal $(59 \mu \mathrm{g} / \mathrm{dL})$, while the serum ferritin level was high $(328 \mathrm{ng} / \mathrm{mL})$. Therefore, we considered the anemia to be due to hemorrhage and chronic inflammation.

Radiological enteroclysis showed intermittent stenosis and lesions suspicious of longitudinal ulcer scars in the distal ileum (Fig. 2). Although pathological examinations of the colon performed at the former hospital and our facility did not reveal any signs of granuloma formation, the patient was diagnosed with $\mathrm{CD}$ based on the characteristic radiographic and endoscopic findings (Fig. 1B, C). She did not exhibit any complications of perianal lesions, intraperitoneal abscesses, apparent fistulas or active uveitis. However, she did display polyarthritis and erythematous lesions, likely extraintestinal complications of CD.

In our hospital, we continued the administration of mesalazine at a dose of $3 \mathrm{~g} /$ day; however, the patient continued to complain of fever and polyarthralgia. We therefore started treatment with ADA at an initial dose of $160 \mathrm{mg}$, followed by $80 \mathrm{mg}$ after two weeks then $40 \mathrm{mg}$ every other week for maintenance therapy. The Crohn's Disease Activity Index (CDAI), one of the most commonly used scales to evaluate the activity of $\mathrm{CD}(8)$, at the time of initiation of ADA treatment was 275 (remission: $\leq 150$, mild activity: 151-220, 


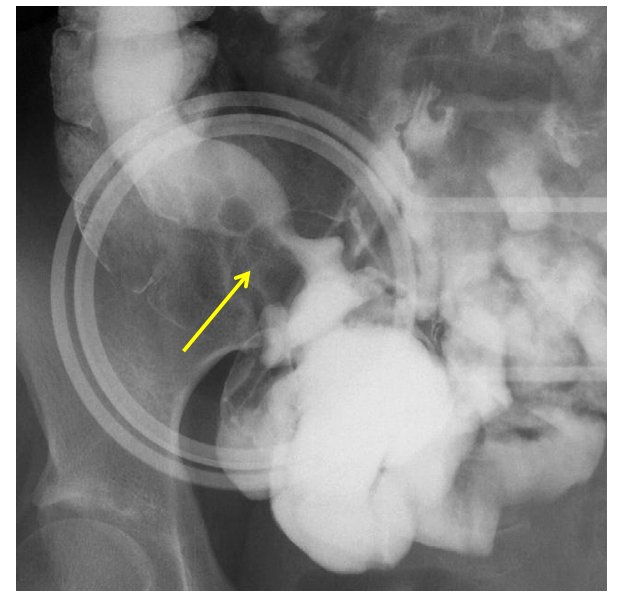

Figure 2. Radiological enteroclysis showed intermittent stenosis in the distal ileum extending approximately $7 \mathrm{~cm}$ from the ileocecal valve (arrow). Longitudinal ulcer scarring was also suspected.

moderate activity: 221-450, severe activity: $>450$ ). Three days after the start of ADA, the polyarthralgia markedly improved. Moreover, the frequency of defecation decreased, reaching an incidence of three bowel movements daily five days after the initiation of ADA treatment. Soon thereafter, the serum transaminase level gradually increased. We suspected a diagnosis of drug-induced liver dysfunction and stopped the dose of mesalazine on day 8 after the start of ADA therapy. Consequently, the serum transaminase level decreased, suggesting that the abnormality in this parameter was indeed drug induced. The patient's clinical symptoms did not worsen after stopping mesalazine, and the CDAI decreased, resulting in clinical remission (CDAI <150). Furthermore, the serum CRP level normalized on day 12 after the initiation of ADA.

However, the patient again complained of abdominal pain and a low-grade fever after the fourth administration of ADA. Simultaneously, the serum CRP level rose to 5.71 $\mathrm{mg} / \mathrm{dL}$, although the frequency of defecation remained at approximately once daily and no diarrhea was noted. At that time, the CDAI continued to be $<150$, and she exhibited no symptoms suggestive of perianal complications. These symptoms and laboratory data are atypical of CD relapse. Therefore, we performed abdominal contrast-enhanced computed tomography (CT), which subsequently revealed thickening of the wall of the thoracoabdominal aorta (Fig. 3B). In contrast, CT did not visualize any active lesions in the intestines or any other abscesses. On a physical examination, we detected an abdominal bruit around the upper abdominal area, which had not been heard at the time of ADA initiation. We thus suspected that the patient had developed aortitis during the course of treatment for CD. Gallium scintigraphy (Fig. 4) and abdominal ultrasonography (Fig. 5A) also showed findings consistent with those of aortitis. Meanwhile, Doppler ultrasonography showed no abnormalities, thus suggesting that the aorta was not stenotic. Finally, no serum myeloperoxidase anti-neutrophil cytoplasmic autoanti- bodies (MPO-ANCA) were detectable, and transthoracic echocardiography showed no abnormal findings, including signs of valvular disorders. Based on the increased serum level of CRP, leukocytosis, abdominal bruit and fever, as well as the CT findings of a thickened thoracoabdominal aorta wall, it is possible that the aortitis was caused by TA.

The ADA therapy was discontinued due to the possibility of its involvement in the development of aortitis. Prior to discontinuation, ADA was administered five times (Fig. 6). We subsequently initiated the administration of oral prednisolone (PSL) at an initial dose of $40 \mathrm{mg}(-1 \mathrm{mg} / \mathrm{kg})$ per day for the aortitis, in accordance with the treatment protocol for TA, and the patient's symptoms and serum CRP level rapidly improved. In addition, the PSL was effective for the aortitis and we therefore tapered the dose according to the following schedule: a daily dose of $40 \mathrm{mg}$ for two weeks followed by $30 \mathrm{mg}$ for one week, $20 \mathrm{mg}$ for four weeks and $15 \mathrm{mg}$ for four weeks. The absence of relapse of the aortitis was confirmed, and we carefully resumed the administration of ADA after tapering the PSL dose to $15 \mathrm{mg} /$ day due to the potential for $\mathrm{CD}$ relapse as a result of stopping ADA and tapering PSL. Furthermore, the patient was unable to receive 5-aminosalicylate as maintenance therapy, as it was thought to be the cause of the drug-induced liver dysfunction. No further exacerbation of the aortitis was noted after resuming the ADA therapy, and the dose of PSL was gradually tapered (a daily dose of $12.5 \mathrm{mg}$ for four weeks followed by $10 \mathrm{mg}$ to the present time). Three months after resuming treatment with ADA, at which time PSL had been used for -5 months, we performed total colonoscopy to evaluate the CD activity, which revealed the disease to be in remission, with mucosal healing (Fig. 1D).

We also performed contrast-enhanced CT approximately 50 days and six months after starting treatment with PSL. Consequently, the thickness of the aortic wall was found to have drastically improved at that time (Fig. 3C). A slight improvement continued to be noted at six months (Fig. 3D), with no worsening associated with tapering the dose of PSL. In addition, abdominal ultrasonography, also performed 50 days after the initiation of PSL, showed a drastic improvement in the aortic wall thickness (Fig. 5B). Currently, the patient has no signs of fever or complaints of diarrhea or abdominal pain, suggesting that the $\mathrm{CD}$ is in clinical and endoscopic remission. Furthermore, the activity of the aortitis remains low. The patient continues to receive PSL at a dose of $10 \mathrm{mg} /$ day continuously in addition to ADA in order to maintain a low activity of the aortitis and CD remission, respectively.

\section{Discussion}

This case was a rare case of $\mathrm{CD}$ in which the patient developed aortitis during treatment with ADA. ADA is used to treat several autoimmune disorders, including TA; however, this case remains rare in terms of the development of aortitis while under ADA therapy. After starting the administration 

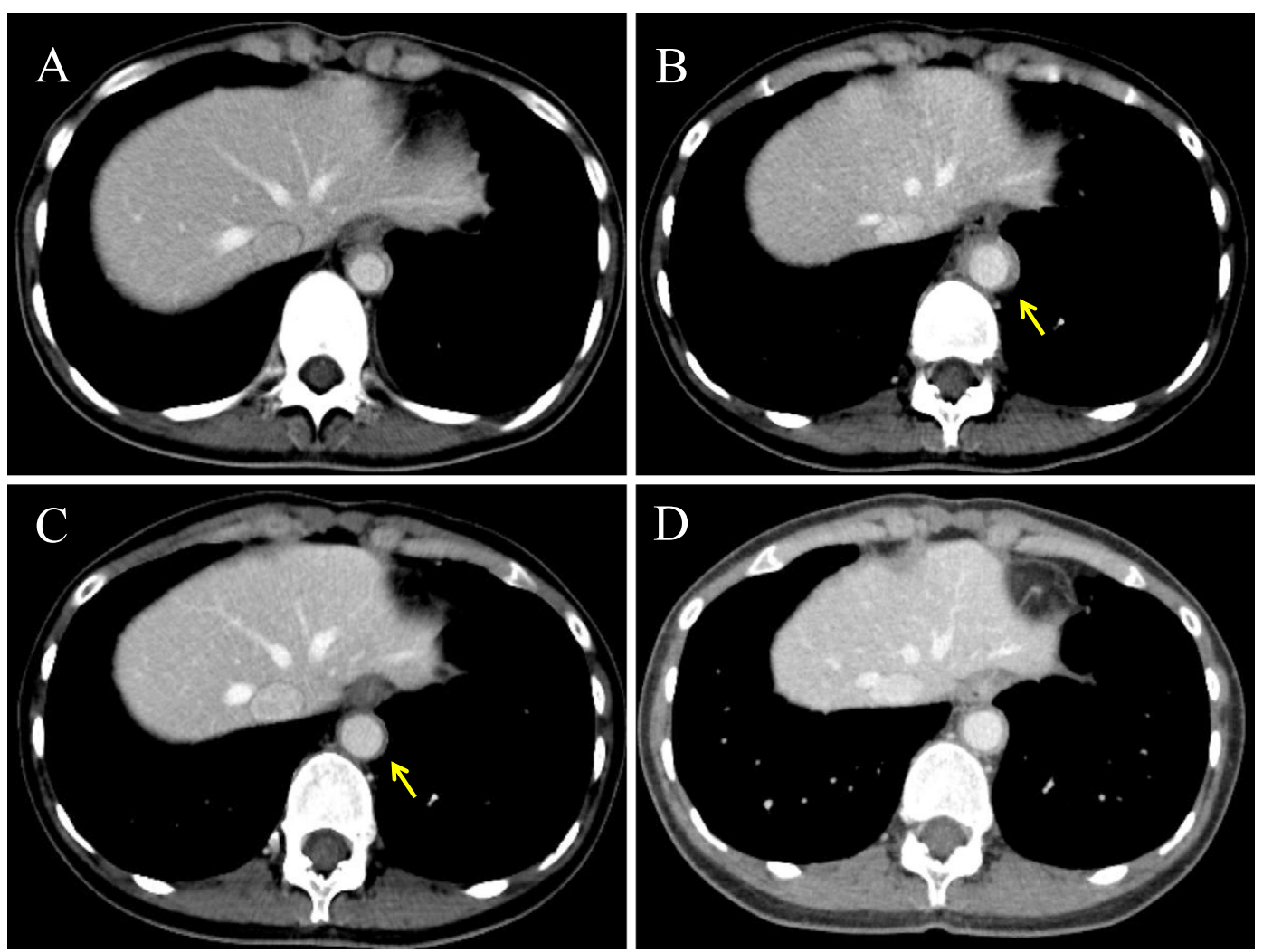

Figure 3. Contrast-enhanced CT showed thickening of the wall of the thoracoabdominal aorta. (A) in retrospect, slight thickening of the aortic wall was already present before starting treatment with ADA. (B) After the fourth administration of ADA, marked thickening of the wall of the thoracoabdominal aorta was noted (arrow). We diagnosed the patient with aortitis. No other abscesses or lesions indicating the origin of the fever were identified. (C) Approximately 50 days after starting treatment with PSL, the thickening of the wall of the aorta drastically improved (arrow). (D) CT performed six months after the initiation of PSL (four months after restarting the ADA therapy). The degree of thickness of the aorta was almost the same as at observed 50 days after the start of PSL.

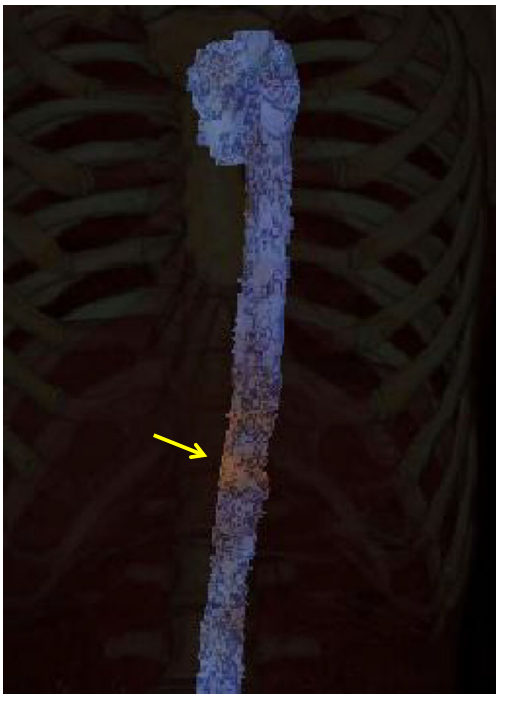

Figure 4. Reconstruction image of gallium scintigraphy. Abnormal accumulation of gallium was detected, distributed from the inferior thoracic aorta to the superior abdominal aorta (arrow). This finding did not restrict the diagnosis to aortitis, although it indicated the presence of active inflammation at this site. Aortitis was the most likely diagnosis. of PSL, the patient's serum CRP level and fever quickly improved, indicating that PSL was effective.

It is possible that the inflammation in this case originated from the relapse of $\mathrm{CD}$ or the associated aortitis, both of which can improve with PSL treatment. However, the frequency of defecation did not increase, and the patient did not complain of diarrhea. Hence, her symptoms differed from those observed at the beginning of the $\mathrm{CD}$ disease course, and we speculate that the inflammation was due to the associated aortitis.

The current patient was diagnosed with $\mathrm{CD}$ based on the detection of longitudinally arranged ulcers suggestive of the disease on colonoscopy. In addition, the findings of radiological enteroclysis were consistent with those of CD. As to the differential diagnosis, patients with Behçet's disease also develop uveitis and vascular lesions. Although the current patient had a past medical history of uveitis, no other manifestations observed in cases of Behçet's disease, such as ulcers of the pudendum or aphthous stomatitis, were noted, and typical deep ulcerations in the ileocecal area were not detected. The characteristic endoscopic finding of intestinal Behçet's disease is the presence of oval, clear, deep, punched-out ulcers in the ileocecal region. In addition, vas- 

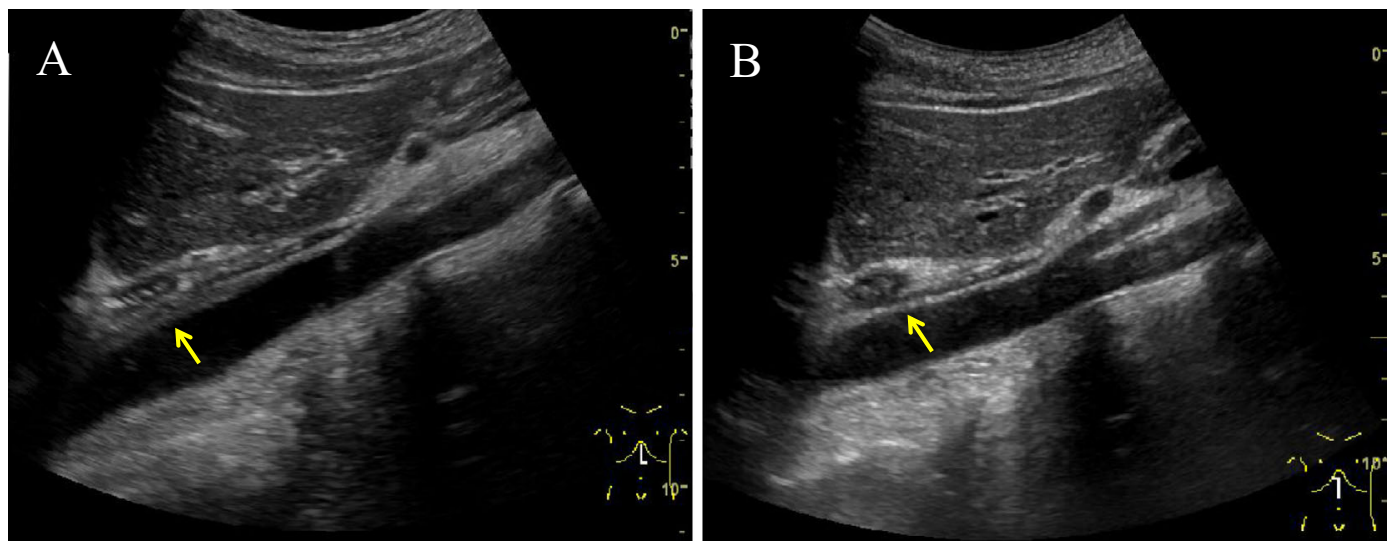

Figure 5. Abdominal ultrasonography was performed before and after starting treatment with PSL. (A) Before starting PSL therapy, the wall of the superior abdominal aorta was thick (arrow); the aortic wall was $5 \mathrm{~mm}$ thick. No abnormal findings were observed on the Doppler study (data not shown). These findings are consistent with a diagnosis of aortitis. (B) Forty days after starting the administration of PSL, the thickening of the wall of the aorta had improved more significantly (arrow).

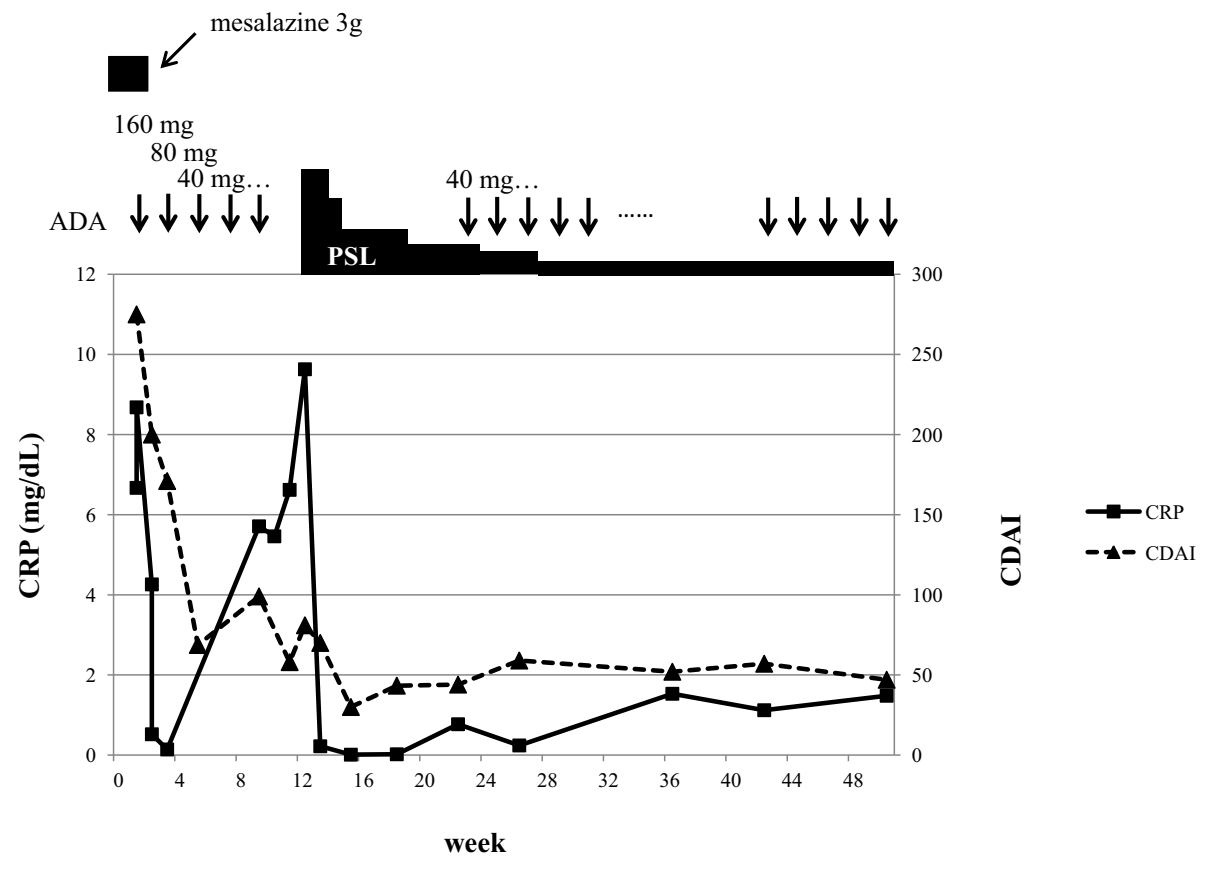

Figure 6. Clinical course of the present patient. The vertical axis shows the serum CRP level and CDAI value. The horizontal axis shows the number of weeks from admission to our hospital. ADA therapy was initiated -2 weeks after admission. The dose of ADA is indicated. When the therapy was restarted, a dose of $40 \mathrm{mg}$ was administered every other week. Treatment with mesalazine was initiated prior to admission, although it was subsequently discontinued -1 week after the initiation of ADA due to the suspicion of drug-induced liver dysfunction.

cular lesions are commonly seen in the veins in patients with vasculo-Behçet's disease, whereas arterial lesions are rare. Furthermore, aortitis may occur in cases of vasculoBehçet's disease, although it is very rare. Therefore, it is difficult to determine whether the lesions observed in the present case were indicative of vasculo-Behçet's disease or intestinal Behçet's disease, and we cannot completely exclude the 'suspicion of Behçet's disease' based on the Japanese criteria for Behçet's disease. Therefore, the likelihood of this differential diagnosis is low. Another differential diagnosis is intestinal manifestations of TA. There are a few reports describing the onset of intestinal ischemia due to TA $(9,10)$. The vasculitis associated with TA occurs in the large vessels, with reported cases involving major branches of the aorta as the responsible lesion, subsequently resulting in extensive intestinal ischemia or necrosis. The longitudinal ulcers in the small intestine detected in this case were located on the opposite side of the mesenterium, consistent 
Table 1. Laboratory Data Obtained before the Start of ADA Therapy.

\begin{tabular}{|c|c|c|c|c|c|c|c|}
\hline & & normal & unit & & & normal & unit \\
\hline TP & 7.3 & $(6.7-8.2)$ & $\mathrm{g} / \mathrm{dL}$ & TIBC & 248 & $(256-407)$ & $\mu \mathrm{g} / \mathrm{dL}$ \\
\hline ALB & 3.1 & $(3.9-5.2)$ & $\mathrm{g} / \mathrm{dL}$ & ferritin & 328 & $(8-129)$ & $\mathrm{ng} / \mathrm{mL}$ \\
\hline T-Bil & 0.2 & $(0.4-1.3)$ & $\mathrm{mg} / \mathrm{dL}$ & & & & \\
\hline AST & 21 & $(10-35)$ & $\mathrm{U} / \mathrm{L}$ & WBC & 7,700 & $(3,500-8,500)$ & $/ \mu \mathrm{L}$ \\
\hline ALT & 12 & $(5-40)$ & $\mathrm{U} / \mathrm{L}$ & $\mathrm{RBC}$ & 3.83 & $(3.7-4.9)$ & $\times 10^{6} / \mu \mathrm{L}$ \\
\hline ALP & 199 & $(100-320)$ & $\mathrm{U} / \mathrm{L}$ & $\mathrm{Hb}$ & 10.2 & $(11.5-15)$ & $\mathrm{g} / \mathrm{dL}$ \\
\hline$\gamma \mathrm{GTP}$ & 19 & $(5-40)$ & $\mathrm{U} / \mathrm{L}$ & Hct & 32.8 & $(35-45)$ & $\%$ \\
\hline $\mathrm{LDH}$ & 152 & $(120-220)$ & $\mathrm{U} / \mathrm{L}$ & $\mathrm{MCV}$ & 86 & $(83-100)$ & $\mathrm{fL}$ \\
\hline $\mathrm{CK}$ & 23 & $(50-170)$ & $\mathrm{U} / \mathrm{L}$ & $\mathrm{MCH}$ & 26.6 & $(28-34)$ & pg \\
\hline AMY & 55 & $(42-121)$ & $\mathrm{U} / \mathrm{L}$ & $\mathrm{MCHC}$ & 31.1 & $(32-36)$ & $\mathrm{g} / \mathrm{dL}$ \\
\hline BUN & 7.0 & $(8-20)$ & $\mathrm{mg} / \mathrm{dL}$ & Reticulocyte & 3 & $(0.5-2)$ & $\%$ \\
\hline $\mathrm{Cr}$ & 0.5 & $(0.4-0.8)$ & $\mathrm{mg} / \mathrm{dL}$ & Platelet & 51.8 & $(15-35)$ & $\times 10^{4} / \mu \mathrm{L}$ \\
\hline $\mathrm{Na}$ & 139.9 & $(136-145)$ & $\mathrm{mEq} / \mathrm{L}$ & & & & \\
\hline $\mathrm{K}$ & 4.7 & $(3.6-4.8)$ & $\mathrm{mEq} / \mathrm{L}$ & QFT & negative & (negative) & \\
\hline $\mathrm{Cl}$ & 100 & $(99-107)$ & $\mathrm{mEq} / \mathrm{L}$ & HBs antigen & negative & (negative) & \\
\hline glucose & 111 & $(80-110)$ & $\mathrm{mg} / \mathrm{dL}$ & HBs antibody & negative & (negative) & \\
\hline HbA1c (NGSP) & 6.0 & $(4.3-5.9)$ & $\%$ & HBc antibody & negative & (negative) & \\
\hline T-Chol & 114 & $(135-240)$ & $\mathrm{mg} / \mathrm{dL}$ & STS (RPR) & negative & (negative) & \\
\hline CRP & 2.47 & $(0-0.35)$ & $\mathrm{mg} / \mathrm{dL}$ & TP antibody & negative & (negative) & \\
\hline $\mathrm{Fe}$ & 59 & $(41-189)$ & $\mu \mathrm{g} / \mathrm{dL}$ & & & & \\
\hline
\end{tabular}

ALB: albumin, ALP: alkaline phosphatase, ALT: alanine aminotransferase, AMY: amylase, AST: aspartate aminotransferase, BUN: blood urea nitrogen, CK: creatine kinase, Cr: creatinine, HbA1c: hemoglobin A1c, HBc: hepatitis B core, HBs: hepatitis B surface, Hct: hematocrit, LDH: lactate dehydrogenase, MCH: mean corpuscular hemoglobin, MCHC: mean corpuscular hemoglobin concentration, QFT: QuantiFERON TB, RBC: red blood cell, STS: serologic test for syphilis, RPR: rapid plasma reagent, T-Bil: total bilirubin, T-Chol: total cholesterol, TIBC: total iron-binding capacity

Table 2. Reported Cases of Associated CD and TA/aortitis Occurring during Treatment with Anti-TNF- $\alpha$ Agents.

\begin{tabular}{|c|c|c|c|c|c|c|c|}
\hline Reference & $\begin{array}{c}\text { Year of } \\
\text { publication }\end{array}$ & $\begin{array}{c}\text { Age at } \\
\text { diagnosis }\end{array}$ & Sex & Type of CD & $\begin{array}{l}\text { Duration of using } \\
\text { anti TNF- } \alpha \text { agent } \\
\text { until occurrence of } \\
\text { TA }\end{array}$ & $\begin{array}{l}\text { Type of anti- } \\
\text { TNF- } \alpha \text { agent } \\
\text { (dose) }\end{array}$ & $\begin{array}{l}\text { Combination } \\
\text { therapies }\end{array}$ \\
\hline 5 & 2011 & 17 & $\mathrm{~F}$ & N.A. & N.A. & IFX $(3 \mathrm{mg} / \mathrm{kg})$ & $\begin{array}{l}\text { Steroid, 5-ASA, } \\
\text { ciprofloxacin }\end{array}$ \\
\hline 6 & 2010 & 20 & $\mathrm{~F}$ & Ileocolonic & 10 months & IFX $(5 \mathrm{mg} / \mathrm{kg})$ & 5-ASA \\
\hline 7 & 2009 & 15 & $\mathrm{~F}$ & Ileocolonic & Several months & IFX (5 mg/kg) & Steroid, AZA \\
\hline 20 & 2008 & 17 & $\mathrm{~F}$ & Colonic & 7 months & IFX (5 mg/kg) & Steroid, 6-MP \\
\hline 21 & 2005 & 27 & $\mathrm{~F}$ & Ileocolonic & N.A. & IFX (N.A.) & $\begin{array}{l}\text { Steroid, } \\
\text { metronidazole }\end{array}$ \\
\hline
\end{tabular}

6-MP: 6-mercaptopurine, AZA: azathioprine, IFX: infliximab, N.A.: not available

with the features of vasculitis, although this is not a specific finding. In the current case, the celiac artery, superior mesenteric artery and inferior mesenteric artery were intact on contrast-enhanced CT, and the disease activity and response to ADA were not parallel between the intestinal lesions and aortitis. Therefore, the intestinal lesions were less likely to be the result of TA, but rather CD.

$\mathrm{CD}$ often exhibits extraintestinal complications, including uveitis, polyarthritis, primary sclerosing cholangitis, erythema nodosum, pyoderma gangrenosum, psoriasis, autoimmune thyroiditis, thromboembolism and vasculitis. Arthri- tis is the most common extraintestinal complication, with $-20 \%$ of CD patients reported to have some form of arthritis. However, the occurrence of vasculitis, particularly in large blood vessels, is a rare complication of $\mathrm{CD}$; TA is a representative example of this. Nevertheless, some cases of CD associated with TA have been reported (1-4).

In the present case, the patient's aortitis met some of the criteria for TA defined in the Japanese Circulation Society guidelines, including fever, an abdominal bruit, increased acute-phase reactions and thickening of the thoracoabdominal aorta wall, as shown on contrast-enhanced CT. Accord- 
ing to the classification criteria of the American College of Rheumatology, two of six criteria, namely an age of $<40$ years and an abdominal bruit, are met in association when the fulfillment of three or more items for the definition of TA (11-13). These criteria include arteriogram abnormalities and occlusion or narrowing of the aorta or its major branches. The current case involved abnormal findings of thickening of the wall of the aorta, but not occlusion or narrowing, on both CT and ultrasonography. Based on these findings, the aortitis could not be diagnosed definitively as TA, but rather possible TA.

In a recent study, anti-TNF- $\alpha$ agents were shown to have a therapeutic effect on TA in addition to CD. To date, $>100$ cases of refractory TA treated with infliximab have been reported, as well as nine cases of ADA (14). Meanwhile, only a few cases of comorbid CD and TA, despite the use of infliximab, have been documented (Table 2). The present case is believed to be the first report of a CD patient who developed aortitis while receiving ADA.

Why did the current patient develop aortitis? In retrospect, slight thickening of the wall of the same part of the aorta was noted on CT before starting ADA therapy (Fig. 3A). Therefore, it is unlikely that the ADA treatment caused the aortitis de novo, although it is possible that it was an exacerbating factor. Another possibility is that the administration of ADA was unable to suppress deterioration of the aortitis without being used in combination with corticosteroids or immunomodulators.

Vasculitis is a rare side effect of treatment with anti-TNF$\alpha$ agents. However, small vessel lesions are more common. Ramos-Casals et al. reported that $72 \%$ of patients who develop vasculitis during anti-TNF- $\alpha$ treatment have concomitant cutaneous lesions $(15,16)$. Furthermore, anti-TNF- $\alpha$ agents may also result in paradoxical effects, such as the development of psoriatic skin lesions (17).

The disease activity of $\mathrm{CD}$ and aortitis were apparently independent of each other in this case. Therefore, it is unlikely that the aortitis was an extraintestinal manifestation of CD. In addition, several types of paradoxical effects have been reported in addition to psoriatic lesions, including vasculitis and arthritis (17). For these reasons, it is necessary to mention the possibility that the vasculitis observed in this case was due to a paradoxical effect.

Another inflammatory bowel disease, ulcerative colitis, is also reported to occur relatively rarely in association with TA. Over 30 cases of concomitant ulcerative colitis and TA have been reported to date (18), and another report demonstrated that $-7 \%$ of patients with TA develop inflammatory bowel diseases, including CD or ulcerative colitis (19).

In the current case, we daringly resumed treatment with ADA in combination with prednisolone, as the administration of prednisolone is inadequate to maintain $\mathrm{CD}$ in remission as maintenance therapy and ADA was very effective for the patient's CD. Although it is not definite that ADA therapy was the exacerbating factor in this case, we should continue to carefully monitor the patient in order to establish whether the aortitis worsens after resuming the dose of ADA. It is very important to clarify whether this patient may successfully use ADA because this medication is very effective for $\mathrm{CD}$ and was a key drug for maintaining $\mathrm{CD}$ remission in this case.

Adding the administration of an immunomodulator was another choice of treatment in this case. However, it is controversial whether immunomodulators should be added to anti-TNF- $\alpha$ agents (22-24), especially in CD patients receiving ADA. The rate of remission was found to be higher when using infliximab in combination with an immunomodulator than without in a prospective study of bio-naïve CD patients (25), although another retrospective study showed no beneficial effects (26). Currently, it is believed that administering immunomodulators in combination with infliximab has beneficial effects in bio-naïve early CD patients. However, there is not enough evidence to confirm the beneficial effects of applying immunomodulators together with ADA. For these reasons, whether the current patient should receive an immunomodulator is unclear. Since the above medications constitute the first treatment regimen for this patient, we initially did not use an immunomodulator concomitantly.

How to properly adjust the dose of PSL is another important issue. As described above, PSL has no effect in maintaining $\mathrm{CD}$ in remission, and we should taper the dose of this drug at this point. However, TA is known to be exacerbated at a high rate when the dose of PSL is reduced. In such cases, immunomodulator therapy is recommended as concomitant treatment. It has been reported that $66-84 \%$ of TA patients require immunosuppressive agents, such as azathioprine or methotrexate, in addition to corticosteroids (27). If the patient's aortitis worsens during the course of tapering PSL, we will consider the concomitant use of an immunomodulator and may be forced to apply PSL at the maintenance dose.

In conclusion, we herein reported a case of $\mathrm{CD}$ in which the patient developed aortitis indicative of TA despite receiving treatment with ADA. This case is significant in terms of the pathology of both CD and TA.

\section{Author's disclosure of potential Conflicts of Interest (COI).}

Tadakazu Hisamatsu: Honoraria, Abbvie and Ajinomoto Pharmaceuticals; Research funding, Abbvie and Ajinomoto Pharmaceuticals.

\section{References}

1. Reny JL, Paul JF, Lefèbvre C, et al. Association of Takayasu's arteritis and Crohn's disease. Results of a study on 44 Takayasu patients and review of the literature. Ann Med Interne (Paris) 154: 85-90, 2003.

2. Farrant M, Mason C, Wong NA, Longman RJ. Takayasu's arteritis following Crohn's disease in a young woman: Any evidence for a common pathogenesis? World J Gastroenterol 14: 4087-4090, 2008.

3. Kusunoki R, Ishihara S, Sato M, et al. Rare case of Takayasu's ar- 
teritis associated with Crohn's disease. Intern Med 50: 1581-1585, 2011.

4. Yilmaz N, Can M, Alibaz-Oner F, Direskeneli H. Clinically silent Crohn's disease in a patient with Takayasu's arteritis unresponsive to conventional therapies. Rheumatol Int 33: 3091-3093, 2013.

5. Osman M, Aaron S, Noga M, Yacyshyn E. Takayasu's arteritis progression on anti TNF biologics: a case series. Clin Rheumatol 30: 703-706, 2011.

6. Katoh N, Kubota M, Shimojima Y, et al. Takayasu's arteritis in a patient with Crohn's disease: an unexpected association during infliximab therapy. Intern Med 49: 179-182, 2010.

7. El-Matary W, Persad R. Takayasu's aortitis and infliximab. J Ped iatr 155: 151, 2009.

8. Best WR, Becktel JM, Singleton JW, Kern F Jr, et al. Development of a Crohn's disease activity index. National Cooperative Crohn's Disease Study. Gastroenterology 70: 439-444, 1976.

9. Simon S, Schittko G, Bösenberg H, Holl-Ulrich K, Schwarz-Eywill M. Fulminant course of a Takayasu's arteritis and rare mesenteric arterial maninfestion. Z Rheumatol 65: 520, 522526, 2006.

10. Cornejo R, Gatica H, Segovia E, Cortés C. Intestinal necrosis as clinical presentation of Takayasu arteritis. Rev Med Chil 130: 1159-1164, 2002 (in Spanish, Abstract in English).

11. Arend WP, Michel BA, Bloch DA, et al. The American College of Rhumatology 1990 criteria for the classification of Takayasu arteritis. Arthritis Rheum 33: 1129-1134, 1990.

12. de Souza AW, de Carvalho JF. Diagnostic and classification criteria of Takayasu arteritis. J Autoimmun 48-49: 79-83, 2014.

13. Jennette JC, Falk RJ, Bacow PA, et al. 2012 revised International Chapel Hill Consensus Conference Nomenclature of Vasculitides. Arthritis Rheum 65: 1-11, 2013.

14. Clifford A, Hoffman GS. Recent advances in the medical management of Takayasu arteritis: an update on use of biologic therapies. Curr Opin Rheumatol 26: 7-15, 2014.

15. Ramos-Casals M, Brito-Zerón P, Cuadrado MJ, Khamashta MA. Vasculitis induced by tumor necrosis factor-targeted therapies. Curr Rheumatol Rep 10: 442-448, 2008.
16. Ramos-Casals M, Brito-Zeron $P$, Muñoz $S$, et al. Autoimmune disease induced by TNF- targeted therapies: analysis of 233 cases. Medicine (Baltimore) 86: 242-251, 2007.

17. Wendling D, Prati C. Paradoxical effects of anti-TNF- $\alpha$ agents in inflammatory diseases. Expert Rev Clin Immunol 10: 159-169, 2014.

18. Kerr GS, Hallahan CW, Giordano J, et al. Takayasu arteritis. Ann Intern Med 20: 919-929, 1994.

19. Ohta Y, Ohya Y, Fujii K, et al. Inflammatory diseases associated with Takayasu's arteritis. Angiology 54: 339-344, 2003.

20. Kellermayer R, Jain AK, Ferry G, et al. Clinical challenges and images in GI. Aortitis as a rare complication of Crohn's disease. Gastroenterology 134: 668-669, 2008.

21. Domènech E, Garcia-Planella E, Olazábal A, et al. Abdominal aortitis associated with Crohn's disease. Dig Dis Sci 50: 11221123, 2005.

22. Alibaz-Oner F, Aydin SZ, Direskeneli H. Advances in the diagnosis, assessment and outcome of Takayasu's arteritis. Clin Rheumatol 32: 541-546, 2013.

23. Calderón R, Estrada S, Ramírez de la Piscina P, et al. Infliximab therapy in a patient with refractory ileocolic Crohn's disease and Takayasu arteritis. Rev Esp Enferm Dig 102: 145-146, 2010 (in English and Spanish).

24. Minami N, Nakase H, Yoshino T, et al. Effect of infliximab on inflammatory bowel disease with Takayasu arteritis: case series and review of the literature. Clin J Gastroenterol 6: 226-230, 2013.

25. Colombel JF, Sandborn WJ, Reinisch W, et al. Infliximab, azathioprine, or combination therapy for Crohn's disease. N Engl J Med 362: 1383-1395, 2010.

26. Drobne D, Bossuyt P, Breynaert C, et al. Withdrawal of immunomodulators after co-treatment does not reduce trough level of infliximab in patients with Crohn's disease. Clin Gastroenterol Hepatol 13: 514-521.e4, 2015.

27. Chatterjee S, Flamm SD, Tan CD, Rodriguez ER. Clinical diagnosis and management of large vessel vasculitis: Takayasu arteritis. Curr Cardiol Rep 16: 499, 2014.

(C) 2015 The Japanese Society of Internal Medicine http://www.naika.or.jp/imonline/index.html 